\title{
Evaluation and management of neurogenic bladder after spinal cord injury: Current practice among physical medicine and rehabilitation specialists in Turkey
}

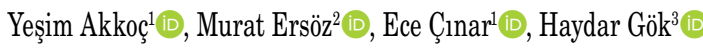 \\ ${ }^{1}$ Department of Physical Medicine and Rehabilitation, Ege University Faculty of Medicine, Izmir, Turkey \\ ${ }^{2}$ Department of Physical Medicine and Rehabilitation, Ankara Yildırım Beyazit University, Ankara, Turkey \\ ${ }^{3}$ Department of Physical Medicine and Rehabilitation, Ankara University Faculty of Medicine, Ankara, Turkey
}

Received: July 09, 2020 Accepted: January 24, 2021 Published online: May 25, 2021

\begin{abstract}
Objectives: This study aims to determine the current trends in evaluation and management of neurogenic bladder secondary to spinal cord injury (SCI) among Physical Medicine and Rehabilitation (PMR) specialists in Turkey.

Materials and methods: Between September 2013 and November 2013, a total of 100 PMR specialists from 18 different provinces of Turkey were included in the study. A 23 -item questionnaire was developed to evaluate the current practice on assessment and follow-up of upper and lower urinary tract dysfunction. The questionnaire was delivered via e-mail to the participants routinely providing care for patients with SCI and all responses were obtained electronically.

Results: For surveillance of the upper urinary tract dysfunction, 93\% of the participants preferred ultrasonography. A total of 59\% of the participants favored an annual assessment and $36 \%$ preferred six-month intervals. Multichannel urodynamics, voiding cystourethrography combined with urodynamics, and video-urodynamics were preferred by $62 \%, 25 \%$, and $10 \%$ of the participants, respectively for surveillance of the lower urinary tract. Urodynamic evaluation was performed annually by $51 \%$ of the participants. In patients with detrusor overactivity unresponsive to the combination of intermittent catheterization (IC) and anticholinergic agents, $66 \%$ preferred to increase the dose and $22 \%$ preferred to switch to another medication. For treatment of areflexic bladder, $78 \%$ preferred IC and $12 \%$ preferred the Credé or Valsalva maneuvers. Treatment of asymptomatic bacteriuria was not favored in patients on IC and indwelling urethral catheter by $33 \%$ and $44 \%$ of the participants respectively. Totally, $84 \%$ participants preferred to administer antibiotics for 10 to 14 days for the treatment of symptomatic urinary tract infection.

Conclusion: Our study results indicate that there are some differences in the current practice of PMR specialists for surveillance and management of SCI patients with neurogenic bladder. These results also emphasize the need for development of guidelines and implementation of continuous medical education activities in this field.
\end{abstract}

Keywords: Neurogenic bladder, spinal cord injury, urologic surveillance.

Spinal cord injury (SCI) is a common cause of neurogenic bladder dysfunction. It can be also caused by other disorders affecting peripheral or central nervous system such as multiple sclerosis, Parkinson's disease, and spina bifida. ${ }^{[1]}$ Neurogenic bladder dysfunction due to SCI poses a significant threat to the wellbeing of patients and quality of life due to its complications including incontinence, urinary tract infection (UTI), stone formation, bladder cancer, and renal impairment. ${ }^{[2,3]}$ The treatment goals for patients with a neurogenic bladder are the preservation of the upper urinary tract, urinary continence, and independence. Although recent guidelines for treatment of neurogenic bladder are available, there is still no consensus on the most optimal surveillance and management options. ${ }^{[4-14]}$

Corresponding author: Ece Çınar, MD. Ege Üniversitesi Tıp Fakültesi Fiziksel Tip ve Rehabilitasyon Anabilim Dalı, 35100 Bornova, İzmir, Türkiye. e-mail: ececinar1@gmail.com 
In Turkey, patients with neurogenic bladder dysfunction are most commonly treated by urologists, Physical Medicine and Rehabilitation (PMR) specialists and gynecologists; however, there is a lack of consensus regarding the ideal treatment and follow-up approach. ${ }^{[15,16]}$ It is of utmost importance to regularly follow SCI patients to avoid life-threatening complications, such as alteration of renal function and infections. Monitoring of renal function and urinary tract problems may be challenging in routine rehabilitation practice. Over time, clinical presentation may change and evolve. Physicians inexperienced in the care of SCI patients with neurogenic bladder may overlook urinary problems and alterations in renal function. Large-volume hospitals and limited time slots may make proper follow-up of these patients more difficult, particularly in the outpatient setting. In the present study, we aimed to determine the current trends in surveillance and management of neurogenic bladder secondary to SCI among PMR specialists in Turkey.

\section{MATERIALS AND METHODS}

This descriptive, cross-sectional study was conducted at Ege University Faculty of Medicine, Department of Physical Medicine and Rehabilitation between September 2013 and November 2013. A total of 100 PMR specialists from 18 different provinces of Turkey were included in the study. All physicians accepting to answer our questionnaire were included in the study. The participants were routinely working with and providing care for patients with SCI. They were reached through the e-mail group of the Turkish Society of Physical Medicine and Rehabilitation (TSPMR). A questionnaire developed by the authors was delivered to PMR specialists (Appendix 1). All responses were received via e-mail. The questionnaire included 23 questions evaluating the current practice on assessment and follow-up of upper and lower urinary tract dysfunction and complications, their optimal frequency and management.A written informed consent was obtained from each participant. The study protocol was approved by the Ege University Faculty of Medicine Ethics Committee (No: 12-11.1/19). The study was conducted in accordance with the principles of the Declaration of Helsinki.

\section{Statistical analysis}

Statistical analysis was performed using the IBM SPSS version 20.0 software (IBM Corp., Armonk, NY, USA). Descriptive data were expressed in mean \pm standard deviation (SD), median (min-max) or number and frequency, where applicable.

\section{RESULTS}

Demographic features of the participants are presented in Table 1. A total of $31 \%$ of the respondents

\begin{tabular}{|lc}
\multicolumn{2}{c}{ TABLE 1 } \\
\multicolumn{2}{|c|}{ Demographic features of the PMR specialists } \\
& Number of participants \% $(\mathrm{n}=100)$ \\
\cline { 2 - 2 } & $\mathrm{n}$ \\
\hline Duration of Physical and Rehabilitation Medicine Practice (years) & 31 \\
$1-5$ & 21 \\
$6-10$ & 15 \\
$11-15$ & 22 \\
$16-20$ & 11 \\
$>20$ & \\
Number of SCI patients with neurogenic bladder seen per month & 42 \\
$1-5$ & 24 \\
$6-10$ & 16 \\
$11-20$ & 8 \\
$21-30$ & 10 \\
$>30$ & \\
Hospital type & 35 \\
University & 2 \\
Charity University & \\
State Training and Research & 51 \\
State & 9 \\
Others & 3 \\
\hline SCI: Spinal cord injury. & \\
\hline
\end{tabular}


were working for one to five years, $21 \%$ for 6 to 10 years, $15 \%$ for 11 to 15 years, $22 \%$ for 16 to 20 years, and $11 \%$ for more than 20 years. When asked how many SCI patients with neurogenic bladder they examined per month, $42 \%$ answered one to five patients, $40 \%$ reported as 6 to 20 patients, and the remaining participants reported as more than 21 patients per month. Totally, $86 \%$ of the respondents were employed by an academic institution (37\% working in a university hospital and $51 \%$ in a state-run research and training hospital). A total of $91 \%$ respondents reported there was an urodynamics laboratory in their hospital.

Of the respondents, $93 \%$ considered that ultrasonography (USG) was the diagnostic tool of choice for routine surveillance of the urinary upper tract, while 7\% favored intravenous urography (IVU) instead of renal USG. Renal scintigraphy and computed tomography were not chosen by any of the respondents (Table 2). Of all physiatrists, $36 \%$ favored follow-up testing every six months, while 59\% and 5\% favored every one and two years, respectively. Renal function testing with creatinine clearance measurement was performed every six months, yearly, and every two years by $39 \%, 23 \%$, and $27 \%$ of the respondents respectively. A total of $11 \%$ of the respondents chose "other" and reported that they performed a routine blood biochemistry at each visit.

\begin{tabular}{|c|c|}
\hline \multicolumn{2}{|c|}{$\begin{array}{c}\text { TABLE } 2 \\
\text { Responses of PMR specialists regarding urinary tract examination }\end{array}$} \\
\hline & \multirow{2}{*}{$\frac{\text { Number of participants } \%(\mathrm{n}=100)}{\mathrm{n}}$} \\
\hline & \\
\hline \multirow{2}{*}{\multicolumn{2}{|c|}{$\begin{array}{l}\text { Evaluation of upper urinary tract } \\
\text { Diagnostic study of choice }\end{array}$}} \\
\hline & \\
\hline USG & 93 \\
\hline IVP & 7 \\
\hline Renal scintigraphy & 0 \\
\hline Renal CT & 0 \\
\hline \multicolumn{2}{|l|}{ Repeat testing } \\
\hline 6 months & 36 \\
\hline 1 year & 59 \\
\hline 2 years & 2 \\
\hline Other & 3 \\
\hline \multicolumn{2}{|l|}{ Frequency of kidney function testing (creatinine clearance) } \\
\hline Every 6 months & 39 \\
\hline Every year & 23 \\
\hline Every 2 years & 27 \\
\hline Other & 11 \\
\hline \multicolumn{2}{|l|}{ Evaluation of lower urinary tract } \\
\hline \multicolumn{2}{|l|}{ Diagnostic study of choice } \\
\hline Multichannel urodynamic study & 62 \\
\hline Videourodynamics & 10 \\
\hline Voiding cystourethrogram & 1 \\
\hline Urodynamic studies plus voiding cystourethrogram & 25 \\
\hline Other & 2 \\
\hline \multicolumn{2}{|l|}{ Repeat testing } \\
\hline 6 months & 29 \\
\hline 1 year & 51 \\
\hline 2 years & 9 \\
\hline Other & 11 \\
\hline \multicolumn{2}{|l|}{ Evaluation of urinary tract infection } \\
\hline \multicolumn{2}{|l|}{ Frequency of urinalysis } \\
\hline 1 month & 18 \\
\hline 3 months & 40 \\
\hline 6 months & 31 \\
\hline 12 months & 5 \\
\hline Other & 6 \\
\hline
\end{tabular}


For surveillance of lower urinary tract, $62 \%$ of the respondents preferred multichannel urodynamic study, $10 \%$ video-urodynamics, and $25 \%$ urodynamic study plus voiding cystourethrogram (Table 2). In addition, $29 \%$ of them favored follow-up testing every six months, while $51 \%$ and $9 \%$ favored every one and two years, respectively.

Urinalysis was performed every month, every three months, every six months, and every 12 months by $18 \%, 40 \%, 31 \%$, and $5 \%$ of the respondents, respectively (Table 2). Asymptomatic bacteriuria was treated by $48 \%$ and $15 \%$ of the respondents in patients on intermittent catheterization (IC) in the presence of pyuria and persistent bacteriuria in the last three urine culture, respectively. Totally, $37 \%$ percent of the respondents did not choose to treat asymptomatic bacteriuria. Symptomatic UTIs were treated for 5, 7, 10, and 14 days by $4 \%, 11 \%, 45 \%$, and $39 \%$ of the respondents, respectively.

Combination of anticholinergic agents and IC was the most optimal option for patients with neurogenic detrusor overactivity. If this option failed, PMR specialists preferred to increase the dose of anticholinergic agent (66\%), change the anticholinergic agent $(28 \%)$, or use botulinum toxin injection to the detrusor muscle (6\%). The IC was the most preferred bladder emptying method for management of areflexic bladder (82\%). Long-term indwelling catheter drainage was favored by $6 \%$ of the PMR specialists and $12 \%$ suggested using Valsalva or Credé' maneuvers to empty the bladder.

All participants were also asked whether they were prescribing anticholinergic drugs to their patients using long-term indwelling catheters. Of the respondents, $34 \%, 21 \%$, and $3 \%$ prescribed anticholinergic drugs only to suprasacral SCI patients, all SCI patients, and only cervical SCI patients, respectively. In addition, $42 \%$ of them did not give any anticholinergic drugs to patients with a long-term indwelling catheter.

Furthermore, $84 \%$ of the respondents felt confident that they appropriately managed their patients, and $16 \%$ thought that they should refer their patients to a tertiary care hospital.

\section{DISCUSSION}

Currently, there are no definitive guidelines for follow-up of neurogenic bladder dysfunction in SCI patients. According to the Paralyzed Veterans of America (PVA) guidelines, a urologic evaluation should be done every year, although there is no consensus among physicians on how frequent this exam should be performed or the range of tests that should be included. ${ }^{[1]}$ The guidelines also state the importance of upper and lower tract evaluations, but do not recommend a special test and followup frequency. Another follow-up regimen is based on a group consensus of the Spinal Cord Injury Think Tank. ${ }^{[2]}$ Accordingly, USG for the evaluation of kidneys and bladder, creatinine clearance for the assessment of renal function, frequency-volume chart and video-urodynamics to define storage/voiding function and, if baseline investigations indicate any renal abnormality, renography (dimercaptosuccinic acid or mercaptoacetyltriglycine) as an optional investigation are recommended in the first three to six months after injury. For ongoing surveillance at six months, 12 months, and then annually, USG (upper tracts, bladder and post-void residual urine volume), creatinine clearance, and serum creatinine measurements at 12 months are recommended. Urodynamic studies are repeated for specific indications, including previous urodynamics showing detrusor-sphincter dyssynergia with sustained elevated vesical pressure or low compliance, recent worsening of symptoms/signs and changing management objectives.

The European Urological Association (EUA) recommends a much more rigorous schedule based on a panel consensus and literature review. ${ }^{[4]}$ Possible UTIs are routinely checked by the patient (dip stick) with urinalysis being done every second month. Upper urinary tract, bladder morphology, and residual urine is examined by USG every six months. Physical examination, blood chemistry, and urine laboratory tests are repeated every year. Detailed investigations are repeated every one to two years and on demand, when the risk factors emerge. The investigation is individualized, according to the patient's actual risk profile, but should in any case include a videourodynamic investigation and should be performed in a neuro-urological center.

All of the above should be more frequent, if the neurological pathology or the neurogenic lower urinary tract dysfunction status demands a closer follow-up. Results of questionnaires on the current practice patterns in urological surveillance and management of SCI patients were first reported in the United States and, later on, in many countries including the United Kingdom, Japan, Canada, Netherlands, France, and Saudi Arabia. ${ }^{[12]}$ 
The questionnaire in the United Kingdom study was performed for SCI units. ${ }^{[6]}$

Monitoring of renal function and detection of deteriorations is of utmost importance. However, it has been reported that $24-\mathrm{h}$ urine collection and creatinine clearance measurement, the most common measurement of renal function in this group of patients, may be misleading due to the decreased muscle mass, disuse, and denervation. ${ }^{[17]}$ Isotopic glomerular filtration rate (GFR) measurement and serum cystatin- $\mathrm{C}$ measurement are recommended as an alternative to creatinine and creatinine clearance measurements. A GFR estimating equation is recommended to derive GFR from serum cystatin rather than serum cystatin concentration alone. This measurement reported to be better than creatininebased calculations in detecting early renal insufficiency in neurogenic patients. ${ }^{[18]}$ On the other hand, these studies are costly and are not readily available in most centers. Mirahmadi et al. ${ }^{[19]}$ suggested using a correction factor of 0.8 in paraplegic and 0.6 in tetraplegic patients after calculating creatinine clearance with the Cockcroft-Gault formula. Serum creatinine clearance remains the most common type of renal function measurement and the current study reflects that majority of rehabilitation professionals in our country perform creatinine clearance measurements every six months or yearly, which is consistent with the aforementioned recommendations.

In a Canadian study, annual routine urodynamic evaluation was favored by $75 \%$ of the urologists and only $11 \%$ of the respondents performed video-urodynamic study on a routine basis. ${ }^{[9]}$ Similarly, $62 \%$ of the PMR specialists preferred annual routine urodynamic evaluation and $10 \%$ preferred video-urodynamic study on a regular basis in the current study. Interestingly, $25 \%$ preferred urodynamic study plus voiding cystourethrogram as a routine yearly evaluation method. Urodynamic study was performed annually or every other year in the Canadian study. ${ }^{[9]}$ The remaining $14 \%$ did not consider a urodynamic study necessary. Similarly, the majority of PMR specialists favored follow-up testing every year in the current study. A considerable number of participants (29\%) preferred follow-up every six months. The majority of the respondents performed a urodynamic examination as frequent as or more frequent than the guideline recommendations.

In the Canadian study, 93\% of the respondents considered that USG was the diagnostic study of choice for routine surveillance of the upper tract, $6 \%$ preferred a yearly IVU, and $1 \%$ chose renal scanning, instead of USG. ${ }^{[9]}$ The current study had similar results: $93 \%$ of the respondents considered that USG was the first-line diagnostic tool for routine surveillance of the urinary upper tract, while 7\% favored IVU. The IC was selected by $93 \%$ of the urologists to manage patients with emptying problem due to neurogenic bladder dysfunction and long-term indwelling catheter drainage was used by $5 \%$ of the urologists in the previous study. ${ }^{[9]}$ In the current study, $78 \%$ preferred IC, $12 \%$ preferred Credé' or Valsalva maneuvers, and 6\% preferred an indwelling catheter. Although individual conditions might have played a role in choosing bladder emptying method, the relatively high frequency of emptying with maneuvers in our study is noteworthy. The IC is the bladder emptying method of choice in most neurogenic bladder patients and majority of the respondents in our study preferred IC over other methods.

In our study, $66 \%$ of the respondents preferred to increase the dose of anticholinergic medications before switching to another molecule for IC patients having still incontinence between catheterizations. Higher doses or a combination of antimuscarinic agents may be an option to maximize the outcomes in neurological patients, although side effects may limit their effectiveness. ${ }^{[20]}$

One of the main limitations to our study is the way questions were delivered. As in all questionnaire studies, response bias is an important tendency which may be caused by the phrasing or answer choices of questions. Although we conducted our study on physicians who are qualified and mostly experienced in care of patients with neurogenic bladder, these results may not perfectly reflect the real-life practice of participants.

The main strength of this study is that we evaluated the frequency of follow-up and type of follow-up and the majority of responses in our study are consistent with the other international studies in which the followup of neurogenic bladder dysfunction is assessed, and with the international guidelines.

In conclusion, our study results indicate that there are some differences in the current practice of PMR specialists for surveillance and management of SCI patients with neurogenic bladder dysfunction. Although treatment regimens may be individualized in response to each patient's individual needs, the results emphasize the need for development of guidelines and implementation of continuous medical education activities in this field. 


\section{Declaration of conflicting interests}

The authors declared no conflicts of interest with respect to the authorship and/or publication of this article.

\section{Funding}

The authors received no financial support for the research and/or authorship of this article.

\section{REFERENCES}

1. Consortium for Spinal Cord Medicine. Bladder management for adults with spinal cord injury: A clinical practice guideline for health-care providers. J Spinal Cord Med 2006;29:527-73.

2. Abrams P, Agarwal M, Drake M, El-Masri W, Fulford S, Reid $S$, et al. A proposed guideline for the urological management of patients with spinal cord injury. BJU Int 2008;101:989-94.

3. Akkoç Y, Ersöz M, Yıldız N, Erhan B, Alaca R, Gök H, et al. Effects of different bladder management methods on the quality of life in patients with traumatic spinal cord injury. Spinal Cord 2013;51:226-31.

4. Stöhrer M, Blok B, Castro-Diaz D, Chartier-Kastler E, Del Popolo G, Kramer G, et al. EAU guidelines on neurogenic lower urinary tract dysfunction. Eur Urol 2009;56:81-8.

5. Canupp KC, Waites KB, DeVivo MJ, Richards JS. Predicting compliance with annual follow-up evaluations in persons with spinal cord injury. Spinal Cord 1997;35:314-9.

6. Bycroft J, Hamid R, Bywater H, Patki P, Craggs M, Shah J. Variation in urological practice amongst spinal injuries units in the UK and Eire. Neurourol Urodyn 2004;23:252-6.

7. Dunn M, Love L, Ravesloot C. Subjective health in spinal cord injury after outpatient healthcare follow-up. Spinal Cord 2000;38:84-91.

8. Kitahara S, Iwatsubo E, Yasuda K, Ushiyama T, Nakai H, Suzuki T, et al. Practice patterns of Japanese physicians in urologic surveillance and management of spinal cord injury patients. Spinal Cord 2006;44:362-8.

9. Blok BF, Karsenty G, Corcos J. Urological surveillance and management of patients with neurogenic bladder: Results of a survey among practicing urologists in Canada. Can J Urol 2006;13:3239-43.

10. Rikken B, Blok BF. Management of neurogenic bladder patients in The Netherlands: Do urologists follow guidelines? Neurourol Urodyn 2008;27:758-62.

11. Denys P, Soler JM, Fatton B, Rischmann P, Yelnik A, Aegerter $\mathrm{P}$, et al. Management of neurogenic bladder patients in France: A survey carried out by the Frenchspeaking neurourology study group (GENULF). Prog Urol 2012;22:540-8.

12. Al Taweel W, Alkhayal A. Neurogenic bladder evaluation and management after spinal cord injury: Current practice among urologists working in Saudi Arabia. Urol Ann 2011;3:24-8.

13. Cameron AP, Lai J, Saigal CS, Clemens JQ; NIDDK Urological Diseases in America Project. Urological surveillance and medical complications after spinal cord injury in the United States. Urology 2015;86:506-10.

14. Linsenmeyer TA, Linsenmeyer MA. Impact of annual urodynamic evaluations on guiding bladder management in individuals with spinal cord injuries. J Spinal Cord Med 2013;36:420-6.

15. Neurogenic Bladder Turkish Research Group, Yıldız N, Akkoç Y, Erhan B, Gündüz B, Yılmaz B, Alaca R, et al. Neurogenic bladder in patients with traumatic spinal cord injury: Treatment and follow-up. Spinal Cord 2014;52:462-7.

16. Yılmaz B, Akkoç Y, Alaca R, Erhan B, Gündüz B, Yıldız N, et al. Intermittent catheterization in patients with traumatic spinal cord injury: Obstacles, worries, level of satisfaction. Spinal Cord 2014;52:826-30.

17. Przydacz M, Chlosta P, Corcos J. Recommendations for urological follow-up of patients with neurogenic bladder secondary to spinal cord injury. Int Urol Nephrol 2018;50:1005-16.

18. Fox JA, Dudley AG, Bates C, Cannon GM Jr. Cystatin $\mathrm{C}$ as a marker of early renal insufficiency in children with congenital neuropathic bladder. J Urol 2014;191(5 Suppl):1602-7.

19. Mirahmadi MK, Byrne C, Barton C, Penera N, Gordon S, Vaziri ND. Prediction of creatinine clearance from serum creatinine in spinal cord injury patients. Paraplegia 1983;21:23-9.

20. Groen J, Pannek J, Castro Diaz D, Del Popolo G, Gross T, Hamid R, et al. Summary of European Association of Urology (EAU) guidelines on neuro-urology. Eur Urol 2016;69:324-33. 
1. State the city you've worked in during the last year.

2. How long have you been a PMR specialist?
a. 1-5 years
b. 6-10 years
c. 11-15 years
d. 16-20 years
e. Longer than 20 years

3. Which setting do you work in?
a. University Hospital
b. Private University Hospital
c. Teaching Hospital
d. State Run Hospital
e. Other (Please explain):

4. How many patients with SCI do you see every month?
a. 1-5
b. 6-10
c. $11-20$
d. 21-30
e. $>30$

5. How do you primarily assess upper urinary tract (UUT)?
a. Ultrasonography
b. IVP
c. Renal scintigraphy
d. CT-abdomen
e. Other (please explain):

6. How often do you routinely repeat UUT assessment (in patients with normal UUTs)
a. Every 6 months
b. Every year
c. Every 2 years
d. Other (please explain):

7. Do you perform creatine clearance measurement ?
a. No
b. Yes, every 6 months
c. Yes, every year
d. Yes, every 2 years
e. Other (please explain):

8. How do you assess the lower urinary tract (LUT)?
a. Urodynamics
b. Video-urodynamics
c. MSUG
d. Urodynamics and MSUG
e. Other (please explain):

9. How often do you routinely repeat LUT assessment (in patients with normal UUTs)
a. Every 6 months
b. Every year
c. Every 2 years
d. Other (please explain):

10. How often do you routinely perform urinalysis and urine culture (in patients without suspected infection)?
a. Every month
b. Every 3 months
c. Every 6 months
d. Every year
e. Other (please explain):

11. Do you treat patients with indwelling catheter and asymptomatic bacteriuria?
a. Yes always
b. No
c. Only if there is pyuria
d. If bacteriuria persists in 3 urine cultures
e. Other (please explain):

12. If you have answered question 11 as yes, for how long do you prescribe antibiotics?
a. 5 days
b. A week
c. 10 days
d. 2 weeks

13. Do you treat patients with asymptomatic bacteriuria and who perform intermittent catheterization?
a. Yes always
b. No
c. Only if there is pyuria
d. If bacteriuria persists in 3 urine cultures
e. Other (please explain):

14. If you have answered question 13 as yes, for how long do you prescribe antibiotics?
a. 5 days
b. A week
c. 10 days
d. 2 weeks

15. How long do you use antibiotics for a symptomatic urinary tract infection?
a. 5 days
b. A week
c. 10 days
d. 2 weeks

16. How often do you advise patients to change indwelling catheters?
a. Every 2 weeks
b. Every 3 weeks
c. Every month
d. Other (please explain):

17. What would be your next treatment approach if a patient on IC and antimuscarinic treatment has high filling pressures and incontinence?
a. Increase the dose of antimuscarinic drug
b. Change with another antimuscarinic
c. Botulinum toxin injections to the detrusor muscle
d. Bladder augmentation surgery
e. Other (please explain):

18. Which method would you prescribe to a patient with areflexic (flaccid) bladder?
a. Intermittent catheterization
b. Credé' or Valsalva maneuvers
c. Indwelling catheter
d. Other (please explain):

19. Does your hospital have urodynamic testing equipment?
a. Yes
b. No 


\section{APPENDIX 1}

Continued

20. If you have answered yes, which clinic or clinics perform the test? (You may mark more than one answer)
a. Physical medicine and rehabilitation
b. Urology
c. Obstetrics and gynecology

21. How often do you perform urodynamic testing to patients with chronic spinal cord injury and neurogenic bladder?
a. On every visit
b. Every 6 months
c. Every year
d. Every 2 years
e. I don't perform urodynamic testing

22. Do you find the care of neurogenic bladder patients in your department satisfactory, or do you refer them to other hospitals?

a. I believe they receive appropriate treatment

b. I often refer patients to other centers

23. Do you prescribe antimuscarinic drugs to patients with long term indwelling catheters?

a. Yes, I prescribe antimuscarinic drugs to all SCI patients

b. Yes, to only those with suprasacral SCI

c. Yes, to only those with cervical SCI

d. No

PMR: Physical Medicine and Rehabilitation; SCI: Spinal cord injury; MSUG: Miction cystouretrography. 